\title{
FATORES PREDITIVOS DE COLEDOCOLITÍASE EM DOENTES COM LITÍASE VESICULAR
}

\author{
Tércio de Campos*, José Gustavo Parreira, André de Moricz, Ronaldo elias Carnut Rego, \\ Rodrigo Altenfelder Silva, Adhemar Monteiro Pacheco Junior \\ Trabalho realizado pelo grupo de vias biliares e pâncreas do Departamento de Cirurgia da Faculdade \\ de Ciências Médicas da Santa Casa de São Paulo, São Paulo, SP.
}

RESUMO - OBjetivo. Identificar fatores clínicos, bioquímicos e ultra-sonográficos preditivos de coledocolitíase no período préoperatório de doentes portadores de litíase vesicular avaliados por colangiografia.

Métodos. Analisamos prospectivamente 148 doentes portadores de litíase vesicular, relacionando critérios pré-operatórios clínicos, bioquímicos e ultra-sonográficos. Todos estes doentes foram submetidos à colangiografia, podendo esta ser endoscópica pré-operatória ou realizada pelo cirurgião durante a colecistectomia. Foram calculados os valores da sensibilidade, especificidade, valores preditivos positivo (VPP) e negativo (VPN), e acurácia para cada um dos critérios estudados, além da correlação de Spearman, Odds ratio e análise de regressão logística para a variável coledocolitíase.

Resultados. Os resultado obtidos mostraram que na análise univariada, a presença de icterícia na internação, a elevação de fosfatase alcalina, gama glutamiltransferase $(\gamma \mathrm{GT})$, aspartato aminotransferase, alanina aminotransferase, bilirrubina total, e ultra-sonografia (USG) com presença de dilatação de vias biliares e visibilização de cálculo na via biliar principal (VBP) tiveram diferença estatisticamente significante para coledocolitíase em nossa amostra $(p<0,00 I) . \mathrm{Na}$ análise de regressão logística, obtivemos como fatores na equação para coledocolitíase: a icterícia na internação, a USG com visibilização de cálculo na VBP e a үGT, nessa ordem. A $\gamma$ GT obteve o maior VPN, sendo considerado o melhor critério isoladamente para se descartar coledocolitíase. Todos os doentes com coledocolitíase em nossa amostra apresentavam ao menos um dos critérios pré-operatórios analisados.

Conclusões: A presença de icterícia e a visibilização de cálculo na VBP foram os melhores fatores para predizer coledocolitíase e a yGT foi o melhor exame para descartar 0 diagnóstico de coledocolitíase.

Unitermos: Coledocolitíase. Colangiografia. Colestase. Icterícia.

\section{INTRODUÇÃO}

A coledocolitíase incide em $8 \%$ a 20\% dos doentes portadores de litíase vesicularl-4. Este achado determina a necessidade de intervenção na via biliar principal (VBP), além da colecistectomia. E a intervenção na VBP poderá ser cirúrgica ou endoscópica,6.

O tratamento endoscópico é indicado no período pré-operatório, no pós-operatório ou eventualmente durante a colecistectomia ${ }^{2}$, enquanto o tratamento cirúrgico é realizado através da exploração pelo ducto cístico ou da coledocotomia clássica, e recentemente também durante a colecistectomia por videolaparoscopia $(\mathrm{VL})^{7}$. Para se estabelecer o tipo de tratamento a ser realizado, é importante o momento em que o diagnóstico de coledocolitíase é feito.

\footnotetext{
* Correspondência

Área I - Vias Biliares e Pâncreas

Departamento de Cirurgia

R. Doutor Cesário Motta Jr., 112 O.M.B - $1^{\circ}$ andar

CEP: 01221-020 - São Paulo - SP

E-mail: cir.pancreas@santacasasp.org.br
}

Quando se estabelece o diagnóstico no período pré-operatório, o tratamento endoscópico, através da colangiopancreatografia retrógrada endoscópica (CPRE) com papilotomia e retirada dos cálculos, é o procedimento de escolha para diversos autores, com colecistectomia complementar2, ${ }^{24,8,9}$. Essa é uma estratégia de tratamento que tem por objetivo simplificar o procedimento operatório ${ }^{8}$.

A preocupação em se fazer o diagnóstico de litíase da VBP no período pré-operatório tornou-se mais importante e tema de vários estudos a partir do desenvolvimento da $\mathrm{VL}$ no final da década de $80^{5}$. Abboud et al..$^{10}$ e Tham et al.' referiram haver maior dificuldade na resolução videolaparoscópica da coledocolitíase.

O achado intra-operatório de coledocolitíase permite ao cirurgião algumas opções. As principais são: exploração cirúrgica das vias biliares por VL (transcística ou por coledocotomia) ou por via aberta", CPRE no intraoperatório ${ }^{2}$, CPRE no pós-operatório, 912-14 ou, ainda, observação do doente, esperando que o cálculo passe espontaneamente pela papila, quando este for menor que $4 \mathrm{~mm}^{14}$.
Discute-se também se é vantagem o achado de cálculos insuspeitos, que são pouco freqüentes, ocorrendo em $1 \%$ a $5 \%$ dos doentes $^{13}$, e que podem ter migração espontânea para o duodeno ${ }^{12}$. Changchien et al. ${ }^{15}$ afirmaram que doentes sem dados clínicos sugestivos, com fosfatase alcalina (FA) e a gama glutamiltransferase $(\gamma \mathrm{GT})$ normais e ausência de alterações da via biliar na ultra-sonografia (USG), apresentam probabilidade menor que $2 \%$ de ter coledocolitíase. Além disso, na maioria das vezes, esses doentes permanecem completamente assintomáticos ${ }^{13,16}$. Com estes argumentos, o emprego da colangiografia intra-operatória $(\mathrm{ClO})$ seletiva passou a ser proposto na literatura por alguns autores ${ }^{16,17}$.

Franceschi et al. ${ }^{18} \mathrm{e}$ Liu et al. ${ }^{3}$ basearam-se em critérios clínicos, laboratoriais e ultrasonográficos na investigação pré-operatória para estabelecer o diagnóstico de coledocolitíase. Liu et al. ${ }^{3}$ referiram que a associação dos critérios clínicos, laboratoriais e ultrasonográficos determinam sensibilidade de 96\% a 98\% para o diagnóstico de coledocolitíase. A análise desses fatores no período pré-operatório poderia determinar se um 
doente teria uma probabilidade baixa, moderada ou alta de apresentar coledocolitíase em associação à litíase vesicular no período préoperatório ${ }^{19}$, permitindo um melhor planejamento terapêutico.

O objetivo deste estudo é identificar fatores clínicos, bioquímicos e ultra-sonográficos preditivos de coledocolitíase no período préoperatório de doentes portadores de litíase vesicular, que foram avaliados por colangiografia (CPRE e ClO).

\section{Métodos}

Foram estudados prospectivamente doentes com diagnóstico de litíase vesicular sintomática submetidos à colecistectomia eletiva, internados no Departamento de Cirurgia da Faculdade de Ciências Médicas da Santa Casa de São Paulo (FCMSCSP), entre setembro de 1998 e setembro de 1999.

Excluíram-se do estudo os doentes que não foram submetidos à colangiografia (oito doentes) ou quando esta foi considerada de qualidade insatisfatória (dez doentes).

Analisaram-se dados de 148 doentes, com média de idade de 48,6 anos ( 15 - 85 anos). Eram $125(84,5 \%)$ do sexo feminino.

Com relação aos sintomas, 129 doentes $(87,2 \%)$ apresentavam queixa de dor em hipocôndrio direito; 18 doentes (12,2\%) referiam dispepsia caracterizada principalmente por empachamento pós-prandial e dor epigástrica em queimação; enquanto que um doente $(0,6 \%)$ apresentava dor abdominal difusa após a alimentação.

Em 65 doentes (43,9\%), encontrou-se algum tipo de doença associada, com a hipertensão arterial presente em 47 doentes $(31,8 \%)$, enquanto que $18(12,2 \%)$ eram diabéticos.

Todos os doentes tinham litíase vesicular diagnosticada pela USG abdominal. Setenta e cinco doentes $(50,7 \%)$ foram operados por laparotomia e 73 (49,3\%) pela via laparoscópica. Houve necessidade de conversão da cirurgia laparoscópica para a cirurgia aberta em dez doentes (13,7\%).

Todos os doentes foram submetidos à colangiografia, podendo esta ter sido realizada tanto no pré-operatório (CPRE) como no intra-operatório $(\mathrm{ClO})$. A escolha foi baseada em dados clínicos, tais como presença ou antecedente de icterícia, antecedente de colangite ou pancreatite, critérios bioquímicos, tais como elevação de enzimas canaliculares (FA e $\gamma \mathrm{GT}$ ) e critérios ultra-sonográficos, tais como dilatação de vias biliares ou visibilização de cálculo na VBP. O doente que apresentava dois ou mais destes critérios alterados era submetido à CPRE pré-operatória, enquanto que aqueles que apresentavam um ou nenhum critério alterado, teriam a $\mathrm{ClO}$ como o procedimento de avaliação da VBP. Este protocolo de estudo é o mesmo protocolo de diagnóstico e tratamento que já era empregado sistematicamente na área de vias biliares e pâncreas da FCMSCSP.

As variáveis estudadas foram sexo, idade maior ou igual a 65 anos, antecedente de icterícia, icterícia na internação, elevação de bilirrubina total (BT), aspartato aminotransferase (AST), alanina amino transferase (ALT), FA, $\gamma G T$, dilataçãa de vias biliares e visibilização de cálculo na VBP pela USG.

A dosagem de FA e de $\gamma \mathrm{GT}$ foi feita pelo método cinético enzimático, no momento da internação ou próximo a esta. Foram considerados os valores destas enzimas como dentro da normalidade ou como elevados. Para confirmar a presença de icterícia, dosou-se a BT desses doentes. A AST e a ALT também foram dosadas e analisadas como normais ou elevadas.

Todos os doentes foram submetidos à USG de abdome na Santa Casa de São Paulo, exame este realizado ou supervisionado por um assistente do Serviço de Diagnóstico por Imagem. Os dados de interesse na USG, além da presença de litíase vesicular, foram a pesquisa de dilatação de vias biliares e visibilização de cálculo na VBP. Considerou-se como dilatação de vias biliares quando o diâmetro da VBP encontrado era maior do que $5 \mathrm{~mm}$.

A CPRE pré-operatória, quando indicada, era realizada sempre pela mesma equipe do Serviço de Endoscopia da Santa Casa de São Paulo, com videoduodenoscópio Pentax de visão lateral ED - 3410 e/ou de visão frontal EG - 290I (Pentax Precision Instruments Corporation). $O$ exame era feito com o doente sob anestesia geral, com intubação orotraqueal e em decúbito lateral esquerdo. Os dados de valor eram dilatação de vias biliares e presença de cálculo na VBP. A colecistectomia era programada para o dia seguinte ao exame.

A ClO era realizada com ao menos três filmes, e com injeção de $3 \mathrm{ml}, 7 \mathrm{ml}$ e $10 \mathrm{ml}$ de urografina $292^{\circledR}$. Na colecistectomia aberta, feita através de incisão de Kocher, era introduzida e fixada uma sonda uretral número 4 ou 6 pelo ducto cístico.

$\mathrm{Na}$ cirurgia por $\mathrm{VL}$, após dissecção e abertura lateral do ducto cístico, um intra-cath ${ }^{\circledR}$ de adulto era posicionado na via biliar e preso com um clipe.

Os dados foram analisados com o programa estatístico SPSS (Statistical Package for Social Sciences para Windows versão 10.0), considerando-se níveis de significância iguais ou inferiores a $5 \%(p \leq 0,05)$. Foi realizada a descrição da amostra, através de contagens e cálculos percentuais mostrados em tabelasresumo.

Em complemento à descrição amostral, foram calculados os valores da sensibilidade, especificidade, valor preditivo positivo (VPP), valor preditivo negativo (VPN) e acurácia. Para a análise estatística, foram usados os seguintes testes e modelos: análise de correlação de Spearman, para todas as variáveis envolvidas neste estudo, além da análise de Odds ratio. Foi feita ainda uma análise de regressão logística (para a variável coledocolitíase), considerando apenas as variáveis que foram estatisticamente significantes na análise univariada, com o intuito de se obter uma fórmula para o cálculo da probabilidade de um doente apresentar coledocolitíase.

\section{Resultados}

Vinte e três doentes (I5,5\%) em nossa amostra eram portadores de coledocolitíase. Nenhum dos doentes relatou antecedente de colangite ou pancreatite aguda.

Analisando as variáveis estudadas, em oito delas, obtivemos forte correlação com coledocolitíase $(p<0,00 /)$ : icterícia na internação, elevação de BT, elevação de AST, elevação de ALT, elevação de FA, elevação de $\gamma G T$, USG com dilatação de vias biliares e USG com cálculo na VBP (Tabela I).

Pelos critérios de seleção pré-operatória, 17 doentes foram submetidos à CPRE, com achado de coledocolitíase em 15 doentes $(88,2 \%)$. Os outros $|3|$ doentes foram submetidos à $\mathrm{CIO}$, com presença de coledocolitíase em oito casos (6, I\%). Dois pacientes que foram submetidos à coledocotomia após a $\mathrm{CIO}$, devido à imagem sugestiva de cálculo na VBP, não apresentavam coledocolitíase após a 


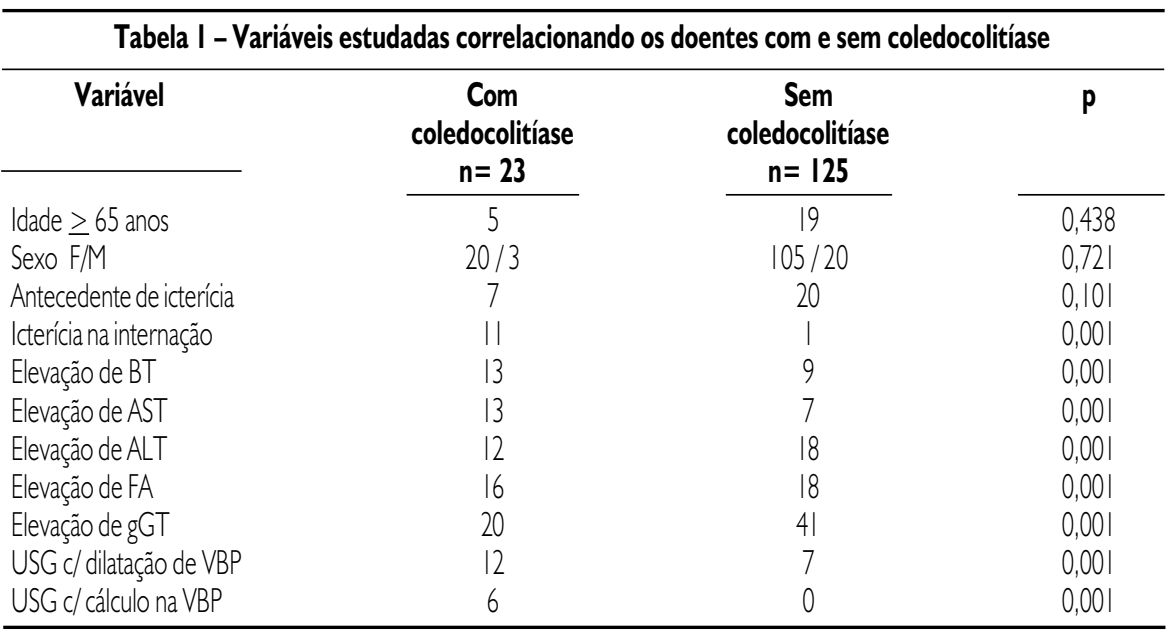

ALT - Alanina aminotransferase; AST - Aspartato aminotransferase; BT - Bilirrubina total; F - Feminino; FA - Fosfatase alcalina; YGT - Gama glutamiltransferase; M - Masculino; USG - Ultra-sonografa; VBP - Via biliar principal.

\begin{tabular}{|c|c|c|c|}
\hline \multicolumn{4}{|c|}{ Tabela 2 - Métodos diagnósticos utilizados e número de doentes com coledocolitíase } \\
\hline Método diagnóstico & Coledocolitíase & Sem coledocolitíase & Total \\
\hline CPRE* & 15 & 2 & 17 \\
\hline $\mathrm{ClO}$ *** & 8 & 123 & $|3|$ \\
\hline Total & 23 & 125 & 148 \\
\hline
\end{tabular}

*Colangiopancreatografia retrógrada endoscópica; **Colangiografia intra-operatória

Tabela 3 - Valores de avaliaçáo com relação à sensibilidade, especificidade, valor preditivo positivo, valor preditivo negativo e acurácia para cada método diagnóstico utilizado

\begin{tabular}{|c|c|c|c|c|c|}
\hline Método diagnóstico & Sensibilidade & Especificidade & VPP & VPN & Acurácia \\
\hline $\begin{array}{l}\text { Icterícia(antecedente) } \\
\text { Icterícia(internação) } \\
\text { FA } \\
\text { YGT } \\
\text { AST } \\
\text { ALT } \\
\text { BT } \\
\text { USG (dilatação) } \\
\text { USG (coledocolitíase) }\end{array}$ & $\begin{array}{c}30,4 \% \\
47,8 \% \\
69,6 \% \\
87 \% \\
56,5 \% \\
52,2 \% \\
56,5 \% \\
52,2 \% \\
26,1 \% \\
\end{array}$ & $\begin{array}{l}84 \% \\
99,2 \% \\
85,6 \% \\
67,2 \% \\
94,4 \% \\
85,6 \% \\
92,8 \% \\
94,4 \% \\
100 \% \\
\end{array}$ & $\begin{array}{l}25,9 \% \\
91,7 \% \\
47,1 \% \\
32,8 \% \\
65 \% \\
40 \% \\
59,1 \% \\
63,2 \% \\
100 \%\end{array}$ & $\begin{array}{l}86,8 \% \\
91,2 \% \\
93,9 \% \\
96,6 \% \\
92,2 \% \\
90,7 \% \\
92,1 \% \\
91,5 \% \\
88 \%\end{array}$ & $\begin{array}{l}75,7 \% \\
91,2 \% \\
83,1 \% \\
70,3 \% \\
88,5 \% \\
80,4 \% \\
87,2 \% \\
87,8 \% \\
88,5 \%\end{array}$ \\
\hline
\end{tabular}

ALT - Alanina aminotransferase; AST - Aspartato aminotransferase; BT - Blilirubina total; FA - Fosfatase alcalina; үGT - Gama glutamiltranfferase;

USG - Ultra-sonografia; VPN - Valor preditivo negativo; VPP - Valor preditivo positivo

exploração. O diagnóstico de coledocolitíase foi feito em 15 doentes $(65,2 \%)$ no pré-operatório pelos critérios utilizados (Tabela 2).

A $\gamma$ GT mostrou a maior sensibilidade (87\%) e o maior VPN (96,6\%). A icterícia na internação e a USG com cálculo na VBP obtiveram maiores especificidade $(99,2 \%$ e $100 \%$, respectivamente) e VPP (91,7\% e $100 \%$, respectivamente). A acurácia da icterícia na internação foi de $91,2 \%$ (Tabela 3).

A probabilidade de um doente apresentar coledocolitíase baseado no VPP de cada variável, em doentes sem antecedente de icterícia e com USG sem alterações das vias biliares, foi de $2,9 \%$. Nos doentes com apenas enzimas canaliculares elevadas, a probabilidade de coledocolitíase variou de $6,7 \%$ a $11,1 \%$, dependendo de qual enzima esteve elevada. Os doentes que não se encontravam ictéricos na internação e cuja USG mostrava dilatação de vias biliares, porém sem cálculos na VBP, a probabilidade de coledocolitíase foi de $40 \%$ a
$42,9 \%$, dependendo se o doente teve ou não antecedente de icterícia, respectivamente. Aqueles doentes que apresentavam icterícia na internação tiveram probabilidade de coledocolitíase de 91,7\%. Quando além da icterícia apresentavam USG com dilatação de vias biliares a probabilidade aumentava para 100\% (Tabela 4).

No cálculo do Odds ratio obtivemos maiores valores para a presença de icterícia na internação ( I |3,7) e para a USG com cálculo na VBP (750) (Tabela 5).

Através do método de regressão logística, foi possível encontrar uma equação capaz de calcular a probabilidade da presença de coledocolitíase baseada nas seguintes variáveis: icterícia na internação, AST, ALT, FA,

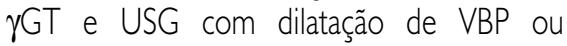
visibilização de cálculo na VBP, e, tal que:

Probabilidade de coledocolitíase $=$ $+\mathrm{e}^{-(-3,689 \mathrm{~A}-8,588 \mathrm{~B}+1,743 \mathrm{C})}$ onde: $A=0$ se anictérico e I se ictérico $B=0$ se USG sem cálculo na via biliar principal e I com cálculo $\mathrm{C}=0$ se $\gamma \mathrm{GT}$ normal e I se elevada $\mathrm{e}=2,718282$

\section{Discussão}

O diagnóstico da coledocolitíase tem importância para estabelecer a conduta nos doentes com litíase vesicular. Estudos foram realizados com o intuito de identificar critérios pré-operatórios para o diagnóstico de coledocolitíase e com isso determinar seu tratamento, porém sem uma conclusão definitiva?.

A colangiografia é o melhor método para se fazer o diagnóstico de coledocolitíase $e^{10,20-22}$, sendo que a CPRE se destaca como a melhor modalidade de colangiografia para o estudo das vias biliares ${ }^{23}$. Em decorrência da CPRE ser um procedimento invasivo, de custo elevado e apresentar taxa de morbi-mortalidade não desprezíve ${ }^{|8,2|}$, outros parâmetros devem ser utilizados na determinação da probabilidade de um doente apresentar coledocolitíase e, assim, indicar seletivamente a CPRE no período pré-operatório 19 .

Liu et al. ${ }^{3}$ relataram como doentes de alto risco para coledocolitíase os que tinham dois critérios positivos entre os clínicos, laboratoriais e ultra-sonográficos, sendo que estes 


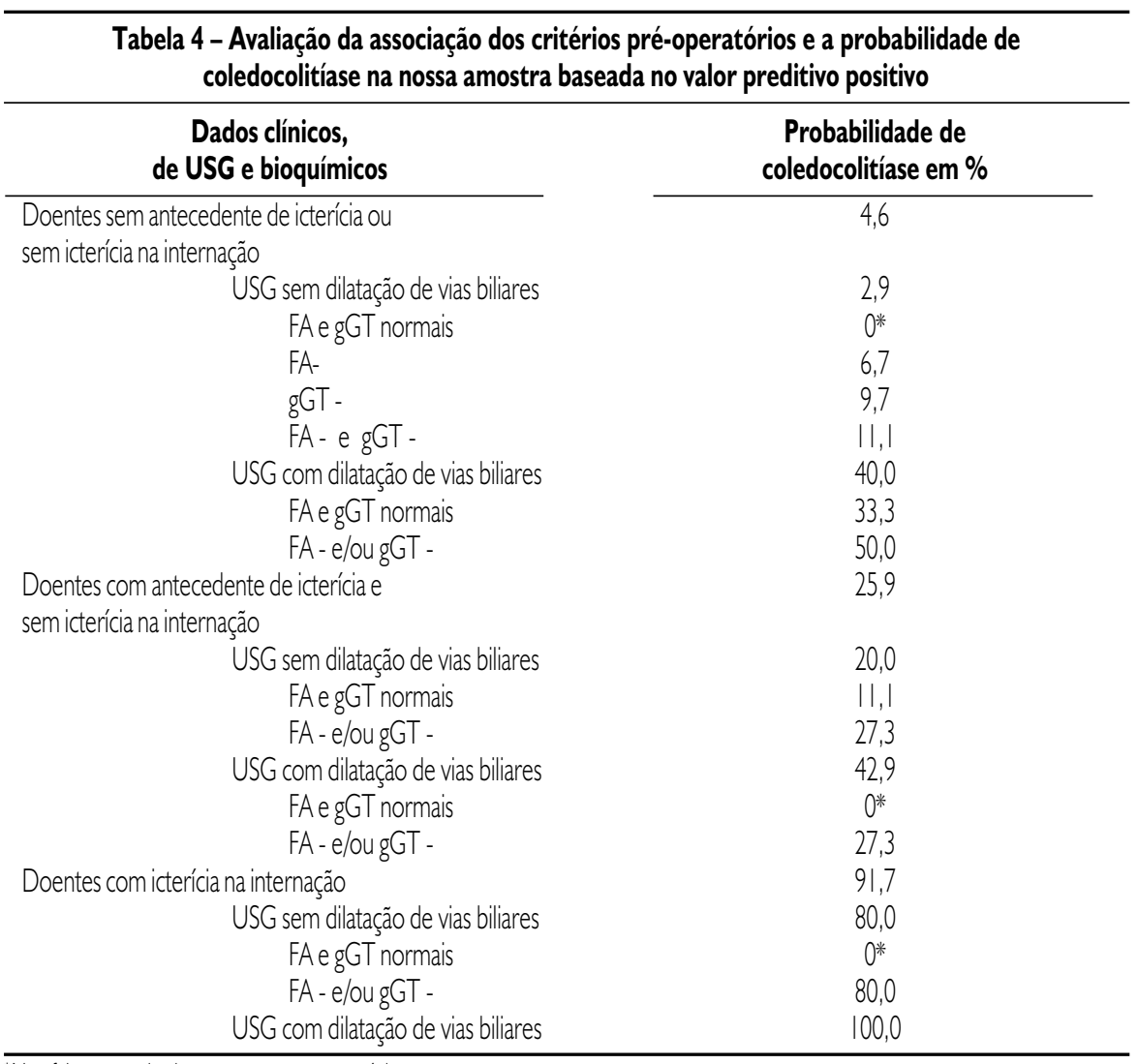

*Não foi encontrado doente com estas características em nossa amostra

$F A=$ Fosfatase alcalina; $\gamma \mathrm{GT}=$ Gama glutamiltransferase; USG = Ultra-sonografia

\section{Tabela 5 - Probabilidade dos doentes apresentarem coledocolitíase baseada} no Odds ratio dos dados pré-operatórios

\begin{tabular}{|c|c|}
\hline Fatores pré-operatórios & Odds ratio \\
\hline Antecedente de icterícia & 2,3 \\
\hline Elevação de ALT & 6,5 \\
\hline Elevação de FA & 13,6 \\
\hline Elevação de $\gamma \mathrm{GT}$ & 13,7 \\
\hline Elevação de BT & 16,8 \\
\hline Dilatação de vias biliares (USG) & 18,4 \\
\hline Elevação de AST & 21,9 \\
\hline Icterícia na internação & $1 \mid 3,7$ \\
\hline Cálculo na VBP (USG) & 750,0 \\
\hline
\end{tabular}

$\overline{\text { ALT }}=$ Alanina aminotransferase; $\mathrm{AST}=$ Aspartato aminotransferase; $\mathrm{BT}=$ = Bilirrubina total; $\mathrm{FA}=$ Fosfatase alcalina; $\gamma \mathrm{GT}$ = Gama glutamiltransferase; USG $=$ Ultra-sonografia

doentes deveriam ser submetidos à CPRE préoperatória. Esses critérios foram empregados no presente estudo.

Os dados clínicos são de grande valor e o fato de o doente encontrar-se ictérico é um fator sugestivo de obstrução da VBP. Em nossa amostra, dos 12 doentes que apresentavam icterícia no momento da internação, apenas um não tinha coledocolitíase. Este doente apresentava elevação de AST e ALT maior que cinco vezes o normal e USG sem dilatação de vias biliares, sugerindo icterícia de origem hepato-celular.

A idade, porém, não mostrou resultado estatisticamente significante, ao contrário do que é visto na literatura ${ }^{10,24}$.

Com relação às enzimas, dos 23 doentes com coledocolitíase, apenas dois (8,7\%) mostravam níveis normais de $\gamma \mathrm{GT}$. Em relação à $F A$, em sete doentes $(34,4 \%)$ os níveis encontravam-se normais, resultados esses semelhantes aos da literatura ${ }^{25}$.

O maior valor preditivo negativo obteve-se para a $\gamma \mathrm{GT}$ com $96,6 \%$, contra $93,9 \%$ da FA, 92,2\% da AST e 90,7\% da ALT. Desse modo, a dosagem de FA é dispensável, pois a $\gamma \mathrm{GT}$ mostrou ser mais importante para descartar coledocolitíase, dado semelhante ao encontrado por Prat et al. ${ }^{26}$.

Em nossa amostra, as enzimas canaliculares não foram importantes para os doentes que se encontravam ictéricos no momento da internação, ou para aqueles que tinham dilatação ou cálculo na VBP pela USG, pois não influenciaram na probabilidade de coledocolitíase neste grupo.

A USG é o melhor método de triagem para detectar alterações da vesícula e vias biliares ${ }^{15,27}$. A USG mostrou dilatação de vias biliares em pouco mais da metade dos doentes com coledocolitíase $(52,2 \%)$, porém todos os doentes em que o ultra-sonografista visibilizou cálculo na VBP, encontrou-se de fato coledocolitíase. $O$ exame foi realizado pelo médico residente com supervisão do assistente, portanto com vários examinadores, o que poderia ocasionar alguma diferença na interpretação dos exames.

Dentre as II variáveis estudadas, oito tiveram uma forte correlação com coledocolitíase $(p<0,00 /)$ : icterícia na internação, elevação de FA, $\gamma \mathrm{GT}, \mathrm{AST}, \mathrm{ALT}, \mathrm{BT}$, dilatação de VBP pela USG e visibilização de cálculo na VBP pela USG. Três variáveis não se mostraram com diferença estatística significativa: idade, sexo e antecedente de icterícia. Dados semelhantes foram obtidos por Hauer-Jensen et al. ${ }^{16}$, apontando dados clínicos, laboratoriais e ultra-sonográficos também com forte correlação com coledocolitíase $(p<0,00 /)$.

A presença de icterícia na internação e a visibilização de cálculo na VBP foram variáveis com melhor VPP, de $91,7 \%$ e 100\% respectivamente.

$\mathrm{Na}$ análise do Odds ratio, duas variáveis contribuíram para um grande aumento da probabilidade de um doente ter coledocolitíase. Observou-se um risco | 13,7 vezes maior de coledocolitíase quando o doente se encontrava ictérico e 750 vezes maior quando a USG visibilizava cálculo na VBP.

Realizou-se ainda uma análise de regressão 


\begin{tabular}{|c|c|c|c|c|}
\hline \multicolumn{5}{|c|}{ Tabela 6 - Grupos com relação à probabilidade de coledocolitíase } \\
\hline & $I^{\circ}$ grupo & $2^{\circ}$ grupo & $3^{\circ}$ grupo & $4^{\circ}$ grupo \\
\hline \multirow[t]{2}{*}{ Características } & $\begin{array}{c}\text { Semicterícia } \\
\text { (prévia ou atual), } \\
\text { USG nle } \\
\text { rGTnl }\end{array}$ & 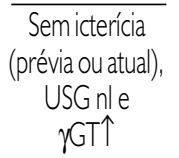 & $\begin{array}{c}\text { A. Sem icterícia atual } \\
\text { e USG c/ } \\
\text { dilataçãa de VBP }\end{array}$ & $\overline{\text { Icterícia atual }}$ \\
\hline & & $\begin{array}{l}\text { ou } \\
\text { Icterícia prévia, } \\
\text { mas não atual, } \\
\text { USG nl e YGt nl }\end{array}$ & 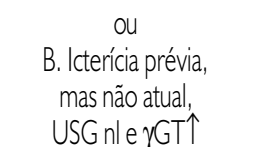 & $\begin{array}{c}\text { e/ou } \\
\text { USG mostrando } \\
\text { cálculo na VBP }\end{array}$ \\
\hline $\begin{array}{l}\text { Probabilidade } \\
\text { de coledocolitíase }\end{array}$ & Baixíssima $(<2 \%)$ & Baixa(2\%-20\%) & Moderada(>20\%-70\%) & $\operatorname{Alta}(>70 \%)$ \\
\hline Proposta & $\begin{array}{c}\text { Sem } \\
\text { colangiografia }\end{array}$ & $\mathrm{ClO}$ & $\begin{array}{c}\text { A. CPRM } \\
\text { B. UE }\end{array}$ & CPRE \\
\hline
\end{tabular}

CIO: Colangiografia intra-operatória; CPRE: Colangiopancreatografia retrógrada endoscópica; CPRM: Colangiopancreatografia por ressonância magnética; $\gamma$ GT: Gama glutamiltransferase; nl: Normal; UE: Ultra-sonografia endoscópica; USG nl: Ausência de dilatação ou cálculos na VBP pela ultra-sonografia; VBP: Via biliar principal; $\uparrow$ : Elevação

logística com objetivo de determinar uma equação para cálculo da probabilidade de coledocolitíase, dentre as variáveis que tiveram significância estatística na análise univariada. Por ordem de importância, a icterícia na internação, a visibilização de cálculo na VBP pela USG e a $\gamma G T$.

Franceschi et al. ${ }^{18}$ citaram como de $4 \%$ a 10\% a incidência de cálculos insuspeitos, ou seja, naqueles doentes sem icterícia, com enzimas canaliculares normais e USG sem alterações da via biliar. Changchien et al. ${ }^{15}$ mostraram que a chance de um doente com enzimas normais e USG normal apresentar coledocolitíase em seu estudo é menor que $2 \%$. Outro dado citado por estes autores é que apenas um em cada dez doentes com cálculos insuspeitos da via biliar desenvolvem algum tipo de sintomatologia. Isto justificaria a conduta expectante com relação a esses cálculos.

A conduta mais cautelosa indica que, frente ao achado incidental de coledocolitíase intra-operatória e caso o cirurgião não tenha um serviço de endoscopia disponível, ou se este serviço não tiver um alto índice de sucesso na resolução de coledocolitíase, deve-se explorar a via biliar no mesmo tempo, seja esta exploração por via laparoscópica ou por via aberta.

Devido a esta preocupação no diagnóstico pré-operatório preciso de coledocolitíase, outros exames foram desenvolvidos na última década para auxiliar nesse diagnóstico, dentre eles a ultra-sonografia endoscópica (UE), a colangiopancreatografia por ressonância magnética (CPRM) a tomografia computadorizada helicoidal (TCH), e a ultra-sonografia intraoperatória $(\mathrm{UIO})^{28}$.

Contudo, um fator de importância que se deve considerar em nosso meio para a definição de um algoritmo ou de uma conduta é o custo de cada exame. Nos Estados Unidos, a CPRE tem um custo estimado de U\$940 quando realizada para diagnóstico e U\$ 1060 para o tratamento da coledocolitíase. A UE por sua vez, tem custo estimado de $U \$ 680$ e a CPRM de U\$ $410^{23}$.

Procura-se avaliar se com este estudo poder-se-ia prever a coledocolitíase. E, além disso, se naqueles doentes com enzimas normais, USG sem alterações da VBP e sem dados clínicos que sugiram coledocolitíase, nos quais a chance de ter coledocolitíase é menor que $2 \%{ }^{15}$, justificar-se-ia a realização de $\mathrm{ClO}$ sistemática. E, ainda, se nos doentes com risco moderado de coledocolitíase poder-se-ia empregar algum destes novos métodos para o diagnóstico de coledocolitíase.

Considerando a seleção pré-operatória feita neste estudo, identificaram-se 15 doentes $(65,2 \%)$ da população com coledocolitíase no pré-operatório. Por outro lado, apenas dois doentes $(11,8 \%)$ que foram submetidos à CPRE não tinham cálculos na VBP.

Na presente casuística, os critérios clínicos, laboratoriais e ultra-sonográficos foram suficientes para indicar a realização de colangiografia seletiva neste grupo de doentes (CPRE ou $\mathrm{ClO}$ ) e, sendo assim, identificar todos os casos de coledocolitíase na nossa amostra. Não houve nenhum doente com coledocolitíase que não apresentasse algum fator que indicasse a sua presença. Deste modo, com os resultados desta amostra, pode-se preferir o emprego seletivo da $\mathrm{ClO}$, opinião esta também compartilhada por outros autores $9,12,16,17,19$.

A possibilidade da passagem de cálculos para a VBP durante a colecistectomia é uma ocorrência que seria evitada com a $\mathrm{ClO}$ sistemática, justificando assim seu emprego. $\mathrm{O}$ argumento contrário sugere que além do problema da $\mathrm{ClO}$ com resultado falso-positivo, o que obrigaria em alguns casos uma exploração desnecessária da via biliar, existe ainda o fato de que se um cálculo passa por um ducto cístico fino, com uma probabilidade muito grande, passará pela papila.

Deve-se ressaltar que, em hospitais-escola e na formação do cirurgião laparoscópico, a $\mathrm{ClO}$ auxilia na identificação precoce de uma eventual lesão de via biliar ${ }^{29}$. Nestas condições de aprendizado do método, observa-se um aumento da incidência deste tipo de lesão, quando comparado com os doentes operados pela via aberta, podendo a $\mathrm{ClO}$ sistemática ser recomendada com o objetivo de evitar lesões graves da VBP ${ }^{30}$. Bresciani ${ }^{20}$ estudou I I 00 doentes que foram submetidos à colecistectomia por VL com realização de $\mathrm{ClO}$ sistemática, encontrando variação anatômica das vias biliares em 12,2\% dos casos, o que poderia facilitar a ocorrência de uma lesão da VBP.

Várias são as observações e análises que se pode fazer frente aos resultados obtidos em nossa amostra. A mais interessante, que demanda maior discussão e demonstra maior aplicabilidade clínica, é a de correlacionar os dados pré-operatórios e selecionar os doentes com probabilidade de apresentar coledocolitíase e, assim, estabelecer um planejamento terapêutico mais adequado.

Alguns autores tentaram dividir esses doentes em três grupos com relação à probabilidade baixa, moderada e alta de coledocolitíase, para, desta forma, orientar a conduta ${ }^{19,26}$

Analisando os dados de probabilidade com o VPP e considerando que a $\gamma$ GT foi o melhor dado bioquímico obtido, principalmente devido ao elevado VPN, dividimos nossa amostra em quatro grupos, considerando o antecedente ou presença de icterícia, a USG e a dosagem de $\gamma$ GT. A CPRE foi o melhor meio de tratar a coledocolitíase, sendo porém indicada apenas quando houvesse grande probabilidade de 
litíase da VBP, devido ao risco de complicações do procedimento (Tabela 6).

Portanto, os critérios pré-operatórios utilizados foram satisfatórios na pesquisa de uma população com maior risco de coledocolitíase. Não houve, na amostra, necessidade de realizar colangiografia nos doentes com litíase vesicular sem sinais pré-operatórios sugestivos de coledocolitíase.

Assim, poder-se-ia realizar a $\mathrm{ClO}$ seletivamente na pesquisa de coledocolitíase, com a possibilidade ainda de utilizar outros métodos pré-operatórios, tais como a CPRM, a UE e a CPRE, conforme o risco do doente.

\section{Conclusões}

Concluímos que, nas condições de realização do presente estudo, foi possível o diagnóstico de coledocolitíase no pré-operatório em $65 \%$ dos doentes, e que a presença de icterícia e a visibilização de cálculo na VBP foram os melhores fatores para predizer coledocolitíase, enquanto que a $\gamma \mathrm{GT}$ foi o melhor exame para descartar o diagnóstico de coledocolitíase.

\section{Conflito de interesse: não há.}

\section{SUMMARY}

\section{Predictors of Choledocholithiasis IN PATIENTS SUSTAINING GALLSTONES}

BACKGROUND. The aim of this study was to identify clinical, biochemical and ultrasonographic predictors of choledocholithiasis in patients sustaining gallstones assessed by cholangiography.

METHODS. In a prospective study, 148 patients were analyzed regarding clinical, biochemical and ultrasonographic data. All patients underwent cholangiography, either preoperative endoscopic or during cholecystectomy. Each variable was compared between the ones who sustained lithiasis in the biliary tree and the others, in order to find out the predictors of choledocholithiasis. Sensibility, specificity, positive predictive value, negative predictive value (NPV) and accuracy were calculated. Spearman correlation, Odds ratio and logistic regression were employed for the statistical analysis, considering $p<0.05$ as significant.

RESULTS. The variables that showed statistical significance were: presence of jaundice, elevated blood serum levels of alkaline phosphatase, g g/utamyltransferase $(\gamma \in T)$, aspartate aminotransferase, alanine aminotransferase, total bilirrubin, and biliary tract dilatation or choledocholithiasis in the ultrasound. The logistic regression presented an equation capable of predicting the probability of choledocholithiasis based in the variables: jaundice, presence of choledocholithiasis in the ultrasound, and blood levels of $\gamma G T$. The bestoption to exclude the presence of choledocholithiasis was $\gamma G T$, as it held the higher NPV. Every patient with choledocholithiasis in this sample sustained at least one of the preoperative criteria analyzed.

Conclusions. Jaundice and choledocholithiasis at the ultrasound were the best predictors of choledocholithiasis; as well as $\gamma G T$ was the most reliable factor to exclude this diagnosis. [Rev Assoc Med Bras 2004; 50(2): 188-94]

KEY wORDS: Choledocholithiasis. Cholangiography. Cholestasis. Jaundice.

\section{REFERÊNCIAS}

I. Tham TCK, Collins JSA, Watson RGP, Ellis PK, Mcllrath EM. Diagnosis of common bile duct stones by intravenous cholangiography: prediction by ultrasound and liver function tests compared with endoscopic retrograde cholangiography. Gastrointest Endosc 1996; 44: I 58-63.

2. Basso N, Pizzuto G, Surgo D, Materia A, Silecchia G, Fantini A, et al. Laparoscopic cholecystectomy and intraoperative endoscopic sphincterotomy in the treatment of cholecysto-choledocholithiasis. Gastrointest Endosc 1999; 50:532-5.

3. Liu TH, Consorti ET, Kawashima A, Ernst RD, Black CT, Greger PH, et al. The efficacy of magnetic resonance cholangiography for the evaluation of patients with suspected choledocholithiasis before laparoscopic cholecystectomy. Am J Surg 1999; 178:480-4.

4. Menezes N, Marson LP, Debeaux AC, Muir $I M$, Auld CD. Prospective analysis of a scoring system to predict choledocholithiasis. Br J Surg 2000; 87: | |76-8|

5. Barkun NA, Barkun JS, Fried GM, Ghitulescu G, Steinmetz O, Pham C, et al. Useful predictors of bile duct stones in patients undergoing laparoscopic cholecystectomy. Ann Surg 1994; 220:32-9.

6. Suc B, Escat J, Cherqui D, Fourtanier G, Hay J, Fingerhut $A$, et al. Surgery vs endoscopy as primary treatment in symptomatic patients with suspected common bile duct stones. Arch Surg 1998; 133:702-8.

7. Materia A, Pizzuto G, Silecchia G, Fiocca F,
Fantini A, Spaziani E, et al. Sequential endoscopic-laparoscopic treatment of cholecystocholedocholithiasis. Surg Laparosc Endosc 1996; 6:273-7.

8. Rosenthal RJ, Rossi RL, Martin RF. Options and strategies for the management of choledocholithiasis. World J Surg 1998; 22: I I 25-32.

9. Hawasli A, Lloyd L, Cacucci B. Management of choledocholithiasis in the era of laparoscopic surgery. Am Surg 2000; 66: 425-31.

10. Abboud PC, Malet PF, Berlin JA, Staroscik R, Cabana MD, Clarke JR, et al. Predictors of common bile duct stones prior to cholecystectomy: a meta-analysis. Gastrointest Endosc 1996; 44: 450-7.

II. Memon MA, Hassaballa H, Memon MI. Laparoscopic common bile duct exploration: the past, the present and the future. Am J Surg 2000; 179:309-15.

12. Borjeson J, Liu SKM, Jones S, Matolo NM. Selective intraoperative cholangiography during laparoscopic cholecystectomy: how selective? Am Surg 2000; 66:616-8.

13. Hee RV. A problem bile duct stone. Br J Surg 2000; 87:838-9.

14. Hashiba K, Cremer M. The current status of the treatment of common bile duct stones in Brazil: a plea for communication. Gastrointest Endosc 200 I; 54:540-2.

15. Changchien, C.; Chuah, S.; Chiu, K. Is ERCP necessary for symptomatic gallbladder stone patients before laparoscopic cholecystectomy ? Am J Gastroenterol 1995; 90:21 24-7.

16. Hauer-Jensen M, Karesen R, Nygaard K, Solheim K, Amlie EJB, Havig O, et al. Prospective randomized study of routine intraoperative cholangiography during open cholecystectomy: long-term follow-up and multivariate analysis of predictors of choledocholithiasis. Surgery 1993; I 13:3 | 8-23.

17. Singh G, Gupta PC, Sridar G, Katariya RN. Role of selective intra-operative cholangiography during cholecystectomy. Aust N Z J Surg 2000; 70: 106-9.

18. Franceschi D, Brandt C, Margolin D, Szopa B, Ponsky J, Priebe $P$, et al. The management of common bile duct stones in patients undergoing laparoscopic cholecystectomy. Am Surg 1993; 59:525-32.

19. Clair DG, Carr-Locke DL, Becker JM, Brooks DC. Routine cholangiography is not warranted during laparoscopic cholecystectomy. Arch Surg 1993; |28:55।-5.

20. Bresciani CJC. Colangiografia intra-operatória em colecistectomia vídeo- laparoscópica: contribuição ao estudo das variações anatômicas e ao diagnóstico das doenças da via biliar principal [Tese Livre Docência]. São Paulo: Faculdade de Medicina, Universidade de São Paulo; 2000.

21. Varghese JC, Liddell RP, Farrell MA, Murray FE, Osborne H, Lee MJ. Diagnostic accuracy of magnetic resonance cholangiopancreatography and ultrasound compared with direct cholangiography in the detection of choledocholithiasis. Clin Radiol 2000; 55:25-35. 
22. Biffl WL, Moore EE, Offner PJ, Franciose RJ, Burch JM. Routine intraoperative laparoscopic ultrasonography with selective cholangiography reduces bile duct complications during laparoscopic cholecystectomy. J Am Coll Surg 200I; 193:272-80

23. Scheiman JM, Carlos RC, Barnett JL, Elta GH, Nostrant TT, Chey WD. Can endoscopic ultrasound or magnetic resonance cholangiopancreatography replace ERCP in patients with suspected biliary disease? A prospective trial and cost analysis. Am J Gastroenterol 200 I; 96:2900-4.

24. Onken JE, Brazer SR, Eisen GM, Williams DM, Bouras EP, Delong ER, et al. Predicting the presence of choledocholithiasis in patients with symptomatic cholelithiasis. Am J Gastroenterol 1996; 91:762-7.
25. Wang $\mathrm{CH}$, Mo LR, Lin RC, Kuo JY, Chang KK. Rapid diagnosis of choledocholithiasis using biochemical tests in patients undergoing laparoscopic cholecystectomy. Hepatogastroenterology 200 I; 48:6 I8-2I.

26. Prat F, Meduri B, Ducot B, Chiche R, Salimbeni-Bartolini R, Pelletier G. Prediction of common bile duct stones by noninvasive tests. Ann Surg 1999; 229:362-8.

27. Lindsell DRM. The diagnostic accuracy of magnetic resonance cholangiopancreatography (MRCP) and ultrasound compared with direct cholangiography in the detection of choledocholithiasis. Clin Radiol 2000; 55:579

28. Polkowski M, Palucki J, Regula J, Tilszer A, Butruk E. Helical computed tomographic cholangiography versus endosonography for suspected bile duct stones: a prospective blinded study in non-jaundiced patients. Gut 1999; 45:744-9.

29. Yasojima EY, Lopes Filho GJ. Colangiografia peroperatória sistemática em colecistectomia videolaparoscópica. Rev Col Bras Cir 2002; 29:92-8.

30. Berci G, Sackier J, Paz-Partlow M. Routine or selective intraoperative cholangiography during laparoscopic cholecystectomy. Am J Surg 199|; |61:355-60.

Artigo recebido: 09/05/03 Aceito para publicação: 01/10/03

\section{www.ramb.org.br}

A Ramb disponibiliza agora um novo serviço para os nossos colaboradores: 0 envio de artigos pela internet. Através do nosso publicador, que agiliza o fluxo de submissão com mais rapidez $\mathrm{e}$ versatilidade, você poderá enviar seu artigo para publicação na Ramb.

Experimente: é muito mais cômodo, muito mais rápido e você ainda poderá acompanhar on line 0 curso de sua apreciação. 